\title{
Benchtop Carbon Fiber Microelectrode Array Fabrication Toolkit
}

Julianna M. Richie ${ }^{1}$, Paras R. Patel ${ }^{1}$, Elissa J. Welle ${ }^{1}$, Tianshu Dong ${ }^{2}$, Lei Chen ${ }^{3}$, Albert J. Shih ${ }^{2}$, Cynthia A. Chestek $k^{1,4,5,6,7}$

${ }^{1}$ Department of Biomedical Engineering, University of Michigan, Ann Arbor, MI, United States of America

${ }^{2}$ Department of Mechanical Engineering, University of Michigan, Ann Arbor, MI, United States of America

${ }^{3}$ Department of Mechanical Engineering, University of Massachusetts Lowell, Lowell, MA, United States of America

${ }^{4}$ Department of Electrical Engineering and Computer Science, University of Michigan, Ann Arbor, MI, United States of America

${ }^{5}$ Neuroscience Graduate Program, University of Michigan, Ann Arbor, MI, United States of America

${ }^{6}$ Robotics Graduate Program, University of Michigan, Ann Arbor, MI, United States of America

${ }^{7}$ Author to whom any correspondence should be addressed.

\section{E-mail: cchestek@umich.edu}

Keywords: carbon electrodes, neural probes, neural recording, electrode surface modification, benchtop fabrication

\section{Abstract}

Background: Conventional neural probes are primarily fabricated in a cleanroom, requiring the use of multiple expensive and highly specialized tools.

New method: We propose a cleanroom "light" fabrication process of carbon fiber neural electrode arrays that can be learned quickly by an inexperienced cleanroom user. This carbon fiber electrode array fabrication process requires just one cleanroom tool, a parylene-c deposition machine, that can be learned quickly or outsourced to a commercial processing facility at marginal cost. Our fabrication process also includes hand-populating printed circuit boards, insulation, and tip optimization.

Results: The three different tip optimizations explored here (Nd:YAG laser, blowtorch, and UV laser) result in a range of tip geometries and $1 \mathrm{kHz}$ impedances, with blowtorched fibers resulting in the lowest impedance. While previous experiments have proven laser and blowtorch electrode efficacy, this paper also shows UV laser cut fibers can record neural signals in vivo.

Comparison with existing methods: Existing carbon fiber arrays either do not have individuated electrodes in favor of bundles or require cleanroom fabricated guides for population and insulation. The proposed arrays use only tools that can be used at a benchtop for fiber population.

Conclusions: This carbon fiber electrode array fabrication process allows for quick customization of bulk array fabrication at a reduced price compared to commercially available probes.

\section{Introduction}


Much of neuroscience research relies upon recording neural signals using electrophysiology (ePhys). These neural signals are crucial to understanding functions of neural networks and novel medical treatments such as brain machine interfaces [1]-[3]. Research is conducted using easy-toassemble probes or commercially available neural recording electrodes. Neural recording electrodes, unique tools with micron-scale dimensions and fragile materials, require a specialized set of skills and equipment to fabricate. A variety of specialized probes have been developed for specific end uses (e.g. a target region) and experiments. However, experiments must either be designed around currently available commercial probes or a lab must invest in the development of a specialized probe which is a lengthy process. Due to the wide variety of neural research, there is high demand for a versatile ePhys probe [1], [4]-[6]. An ideal ePhys probe would feature a small recording site, low impedance [7], and customizable geometry to adapt to the experimental target regions.

Traditionally, commercial probes are silicon-based due to their ability to be fabricated in large batches with a high recording site density [8], [9] and relatively reliable functional stability [10]-[13]. Silicon arrays typically come in one of two designs: a "bed of needles" (Utah) or a planar shank (NeuroNexus [14], Cambridge Neurotech [15], Neuropixel [16]). If an experiment requires a more specific probe geometry than what is commercially available it would require an intensive design, fabrication, and validation process [4], [17], [18]. However, both commercial designs cause tissue damage [19], [20], continuous inflammation around shank sites, increased immune cell response, and decreased neural body density [20]-[22]. The silicon based electrodes corrode and crack [12], [23], insulation detaches or dissolves[10], [24], and traces break from internal and external stresses [12], [23], [25]. These issues cause signals to be lost either due to the mechanical separation of the electrode from the backend connector or in the resulting noise increase that accompanies these failures. Additionally, commercial probes are expensive to produce. The high cost of these probes limits the number of experiments that can be done at a given time. With cost and biocompatibility issues as deterrents, labs often turn to fabricating their own probes with alternative methods and materials.

Thus, sub-cellular scale neural probes are being developed to improve the biological response. Net10/50 probes [5], [26] and silicon carbide "ultramicroelectrodes" [24] use thin flexible shanks to reduce insertion damage. The probes insert quickly and show reliable recordings. The probes also tend to be more mechanically robust due to the flexible nature of the cable reducing the stresses on the electrode sites compared to a traditional silicon array. It is also easy to create a batch of this type of probes at once. However, these devices require either an additionally fabricated and integrated shuttle [5], [26] or need to be coated with a stiffening agent for insertion [24]. Both options offer low damage insertion with neural spike recording capability, but are not commercially available. Fabrication of either design is very difficult to replicate without specialized cleanroom facilities [5], [27], [28] and experience with fabrication processes.

To avoid the expense and difficulty of manufacturing state-of-the-art devices, many labs make their own microwire arrays. Microwires are customizable at the benchtop with reliable results or can be purchased commercially (e.g. Microprobes for Life Science, Tucker-Davis Technologies), offering low damage insertion, a wide recording area, and a small electrode site. However, microwires deform upon insertion [29], cause tissue damage, and can deviate from their initial target [19], [29]-[31]. Commercial microwires have caused moderate inflammation and encapsulation, degrading the electrode [6], [32], [33]. The microwires may also corrode in vivo, causing cracks in the insulation and electrode sites [30]. In addition, microwire arrays often require a headstage that limits which small animal models can be 
studied to those at least the size of a mouse. Commercial headstages can require additional cleanroom processing to improve back end connectorization [34].

In response to the high cost and biocompatibility issues, carbon fiber electrodes may offer an avenue for neuroscience labs to build their own probes without the need for specialized equipment. Carbon fibers are an alternative recording material with a small form factor that allows for low damage insertion. Carbon fibers provide better biocompatibility and considerably lower scar response than silicon [35]-[37] without the intensive cleanroom processing [29]. Carbon fibers are flexible, durable, easily integrated with other biomaterials [37], and maintain electrode integrity in vivo for months [35], [38]. Despite the many advantages of carbon fibers, many labs find manual fabrication of these arrays to be arduous. Some groups [39] combine carbon fibers into bundles that collectively result in a larger $(\sim 200 \mu \mathrm{m})$ diameter than a traditional microwire [39], [40]. Others have fabricated individuated carbon fiber electrode arrays, though their methods require cleanroom-fabricated carbon fiber guides [41]-[43] and equipment to populate their arrays [35], [42], [43]. To address this, we propose a method of fabricating a carbon fiber array that can be performed at the lab benchtop that allows for impromptu modifications. The resulting array maintains individuated electrode tips without specialized fiber populating tools. Additionally, multiple geometries are presented to match the needs of the research experiment. Building from previous work [35], [41], [44], this paper provides detailed methodologies to build and modify several styles of arrays manually with minimal cleanroom training time needed.

\section{Methods}

\section{Carbon Fiber Array Assembly}

Carbon fiber arrays are composed of three parts: a custom printed circuit board (PCB), a backend connector, and an inexpensive sample of $6.8 \mu \mathrm{m}$ carbon fibers (T-650/35 3K, Cytec Thornel, Woodland Park, NJ). All design files associated with designs presented below are available for download, including three different PCBs: "Flex Array", "Wide Board", and "ZIF" (Figure 1) on the MINT website (https://mint.engin.umich.edu/technology-platforms/carbon-fiber-electrodes/). The population and functionalization of a 16-channel Flex Array build is described in detail (build video in supplemental). Several tip optimization techniques to improve electrophysiological recording will also be discussed. A complete materials list including cost is shown in Table 1, with processing steps explained below.

\begin{tabular}{|c|c|c|c|c|}
\hline MATERIALS & PART NUMBER & DISTRIBUTOR & QTY & $\begin{array}{l}\text { UNIT } \\
\text { COST } \\
\text { (USD) }\end{array}$ \\
\hline Wide Board ${ }^{+}$ & $\mathrm{n} / \mathrm{a}$ & Advanced Circuits & 1 & 3 \\
\hline $\begin{array}{l}\text { Pin Terminal Connector } \\
\text { (Wide Board Only) }\end{array}$ & ED11523-ND & DigiKey & 16 & 10 \\
\hline $\mathrm{ZIF}^{*}$ & $\mathrm{n} / \mathrm{a}$ & $\begin{array}{l}\text { Coast to Coast } \\
\text { Circuits }\end{array}$ & 1 & 9 \\
\hline $\begin{array}{l}\text { Hirose Connector } \\
\text { (ZIF Only) }\end{array}$ & H3859CT-ND & DigiKey & 2 & 2 \\
\hline $\begin{array}{l}\text { TDT Shroud } \\
\text { (ZIF Only) }\end{array}$ & Z3_ZC16SHRD_RSN & TDT & 1 & 3.5 \\
\hline
\end{tabular}




\begin{tabular}{|c|c|c|c|c|}
\hline Flex Array** & $\mathrm{n} / \mathrm{a}$ & MicroConnex & 1 & 68 \\
\hline $\begin{array}{l}\text { Omnetics Connector* } \\
\text { (Flex Array Only) }\end{array}$ & A79024-001 & Omnetics Inc & 1 & 35 \\
\hline $\begin{array}{l}\text { Omnetics Connector* } \\
\text { (Flex Array Only) }\end{array}$ & A79025-001 & Omnetics Inc & 1 & 35 \\
\hline 2 Part Epoxy ${ }^{++}$ & 1FBG8 & Grainger & 1 & 3 \\
\hline Silver Epoxy $(10 z)^{++}$ & $\mathrm{H} 20 \mathrm{E} / 1 \mathrm{OZ}$ & Epoxy Technology & 1 & 125 \\
\hline $\begin{array}{l}\text { UV Epoxy }(8 o z)^{++} \\
\text {(Flex Array Only) }\end{array}$ & OG142-87/8OZ & Epoxy Technology & 1 & 83 \\
\hline $\begin{array}{l}\text { 353ND-T Epoxy (8oz) }{ }^{++} \\
\text {(ZIF and Wide Board Only) }\end{array}$ & 353ND-T/8OZ & Epoxy Technology & 1 & 48 \\
\hline Probe storage box & G2085 & Melmat & 1 & 2 \\
\hline Sodium p-toulenesulphonate $(100 \mathrm{~g})$ & 152536 & Sigma-Aldrich & 1 & 59 \\
\hline 3,4-ethylenedioxythiophene $(25 \mathrm{~g})$ & 096618 & Sigma-Aldrich & 1 & 102 \\
\hline
\end{tabular}

\section{Printed Circuit Boards}

Wide Board, a ZIF based PCB (referred to as 'ZIF' here onwards), and Flex Array PCBs were designed in Eagle CAD (Autodesk, San Rafael, CA). Wide Board and ZIF designs were commercially manufactured (Advanced Circuits, Aurora, CO) and are compatible with Tucker-Davis Technologies (TDT) headstages (Figures $1 \mathrm{~A}$ and $\mathrm{B}$ respectively). Flex Arrays were fabricated at a commercial facility (MicroConnex, Snoqualmie, WA) (Figure 1C). Soldering pad and trace sizing vary between each design (Table 2). Wide Boards are the easiest to fabricate. They have a pitch of $3 \mathrm{~mm}$, exposed trace size of 1.5 $\mathrm{mm} \times 4 \mathrm{~mm}$, and are useful for applications where interelectrode distance doesn't matter, for example, soak testing or testing coating viability. The 16-channel ZIFs have a pitch of $150 \mu \mathrm{m}$ and an exposed trace size of $0.75 \mathrm{~mm} \times 0.07 \mathrm{~mm}$, which is sufficiently small for insertion testing or acute or chronic ePhys recordings. The smallest of these three designs is the 16-channel Flex Array, with an electrode pitch of $132 \mu \mathrm{m}$. Due to the small pitch, two traces are used per fiber to help align the fibers and create a well for the silver epoxy. One fiber per trace is possible (66 $\mu \mathrm{m}$ pitch, for 32-channels) with smaller particle epoxy, but requires a skilled hand to place the epoxy and fiber without shorting the electrodes. 


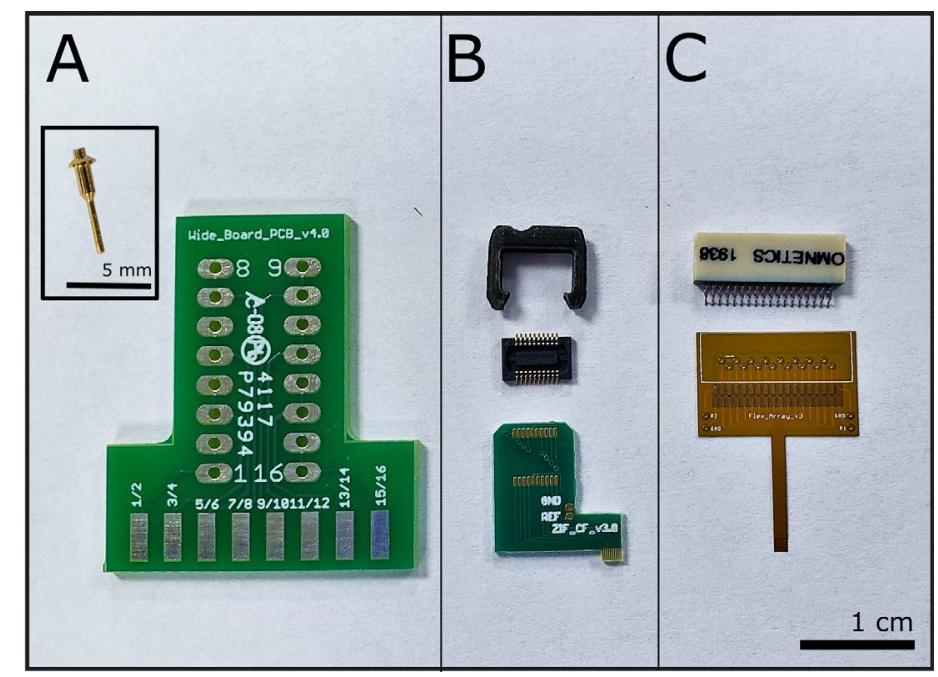

Figure 1: Connectors and associated printed circuit boards. (A) Wide Board with one of sixteen necessary connectors in inset. (B) ZIF and one of two Hirose connectors and TDT shroud. (C) Flex Array with 36-pin Omnetics connector.

\begin{tabular}{|c|c|c|c|c|c|}
\hline PCB NAME & CONNECTOR & $\begin{array}{c}\text { SOLDERING } \\
\text { PAD SIZE } \\
(\mathrm{mm})\end{array}$ & $\begin{array}{c}\text { EXPOSED } \\
\text { TRACE SIZE } \\
(\mathrm{mm})\end{array}$ & $\begin{array}{c}\text { TRACE } \\
\text { PITCH } \\
(\mu \mathrm{m})\end{array}$ & CHANNELS \\
\hline Wide Board & $\begin{array}{c}\text { Mill-Max } \\
9976-0-00-15-00-00-03-0\end{array}$ & $3.25 \times 1.6$ & $1.5 \times 4.0$ & 3000 & 8 \\
\hline ZIF & $\begin{array}{c}\text { Hirose } \\
\text { DF30FC-20DS-0.4V, }\end{array}$ & $0.23 \times 0.7$ & $0.75 \times 0.07$ & 152.4 & 16 \\
\hline Flex Array & Omnetics A79024-001 & $0.4 \times 0.8$ & $0.6 \times 0.033$ & 132 & 16 \\
\hline
\end{tabular}

\section{Soldering Omnetics}

The first step in building any of these devices is soldering the connector. This requires the use of a stereoscope (SMZ445, Nikon Instruments Inc, Melville, NY) and a soldering iron with a fine tip (0.1$0.2 \mathrm{~mm}$ ). For a lab without soldering equipment, this step can be outsourced to any PCB assembly house. Due to the melting temperature of the polyimide board, a soldering iron temperature of $315^{\circ} \mathrm{C}\left(600^{\circ} \mathrm{F}\right)$ was used to reduce the chance of pads separating from the board. Flux was applied to all contacts before a small amount of solder was placed on the back row of pads. Solder mounds had flat tops so the Omnetics pins were able to sit evenly across them (Figure $2 \mathrm{~A}$ ). The two outer-most pins were pushed into the solder with the tip of the iron to secure the connector in place. The remaining pins were secured by pushing the tip of the iron between the front pins and pushing them down (Figure $2 \mathrm{~B}$ ). The front pins were soldered to their respective pads. The remaining flux was cleaned off with $100 \%$ isopropyl alcohol (IPA) rinses and a brush (855-5, MG Chemicals, Canada) with bristles cut down to 5 $\mathrm{mm}$.

To prevent the Omnetics connector from deforming and pulling away from the Flex Array, the connections were insulated using a two-part epoxy (Sy-SS, Super Glue Corporation, Ontario, CA). Epoxy 
was mixed in a dish, pulled into a $1 \mathrm{~mL}$ syringe, excess epoxy was wiped from the tip, and a $23 \mathrm{G}$ needle attached. Epoxy was applied bevel side down against the top of the pins to encase the pins and minimizing air bubbles (Figure $2 \mathrm{C}$ ). A small amount of epoxy was applied to each side of the Omnetics connector on the board to secure the two during future handling steps (Figure $2 \mathrm{D}$ ). Boards were left to cure overnight at room temperature.

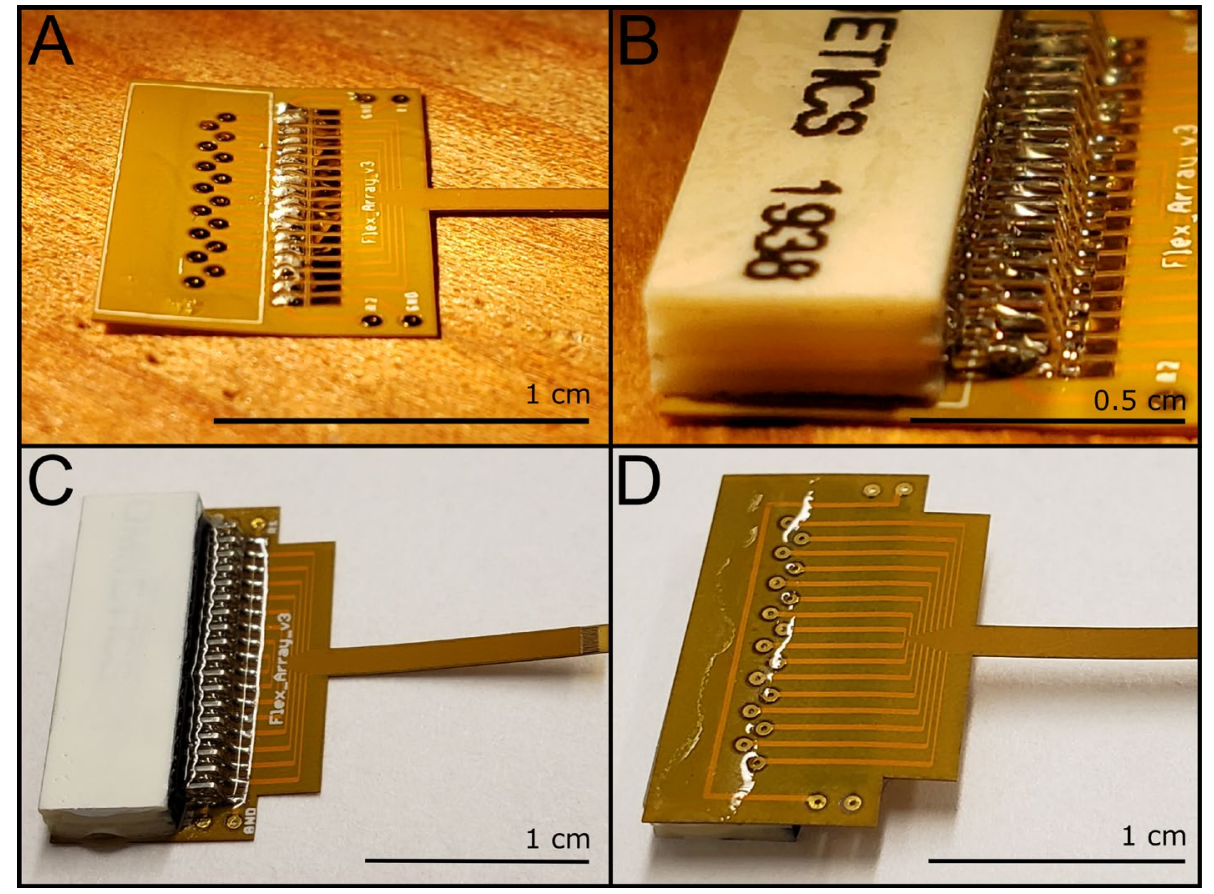

Figure 2: Soldering and insulation steps for the Flex Array. (A) Laying the solder for the bottom Omnetics pins. (B) Back pins secured in place with the front pins ready for soldering. (C) Epoxy insulated Flex Array, note that the epoxy does not cover the reference and ground vias on either side, and (D) Back side of the Flex Array with a band of epoxy across the pad vias (not the ground and reference vias) and wrapped around the side of the board toward the edge of the Omnetics connector.

\section{Fiber Placement and UV epoxy}

The prepared PCB was placed onto putty under the stereoscope (in the video, the putty is placed on a wooden block for ease of movement). Pulled glass capillaries (TW120-3, World Precision Instruments, Sarasota, FL) were made using a glass puller and filament (P-97 and FB315B, Sutter Instrument Co., Novato, CA) under the following settings: Heat=900, Pull=70, Velocity= 35, Time= 200, Pressure $=900$ (numbers are unitless and specific to this device). Pulled capillary tips were cut to easily fit between the traces of the board (Figure 3 A). Silver epoxy (H2OE, Epoxy Technology, Billerica, MA) was mixed in a dish according to manufacturer specifications. The glass capillary tip was dipped into silver epoxy and applied between pairs of adjacent traces (Figure $3 \mathrm{~B}$ ) resulting in 8 pairs of connected traces. Traces are shorted together in this way to ease the manual demand of epoxy and fiber laying, however, one fiber per trace is possible for a practiced user (supplemental).

Fibers were initially cut to 2-4 $\mathrm{mm}$ in length with a straight razor and separated into single fibers. This was accomplished by gently pulling a laminated piece of paper over the top of the carbon fiber bundle. The laminated paper helps to transfer static into the fibers causing them to separate on 
their own. A pair of Teflon coated tweezers (11626-11, Fine Science Tools, Foster City, CA) was used to pick up a single carbon fiber segment. Fibers were placed in the silver epoxy mounds (Figure $3 \mathrm{C}$ ). $\mathrm{A}$ clean capillary was used to adjust the fibers, so they were perpendicular to the end of the board, parallel to the length of the board, and buried beneath the epoxy (Figure $3 \mathrm{D}$ ). Carbon fibers were kept clean of silver epoxy past the edge of the board. Arrays were placed on a wooden block without putty, with the carbon fiber portion stick over the edge, and then put into an oven for 20 minutes at $140{ }^{\circ} \mathrm{C}$ to cure the epoxy. The wooden block allows for easy transport of the device in and out of the oven, while also holding no static charge that could deform the carbon fibers' placement. The technique was repeated on the backside of the array resulting in a 16-fiber array. After curing, traces were visually inspected to ensure the connections had no shorts between fibers. Any epoxy shorts or spills were removed with a clean pulled glass capillary. A practiced user can achieve placement angles that are within 0.35 degrees for all fibers perpendicular to the edge of the board [41].

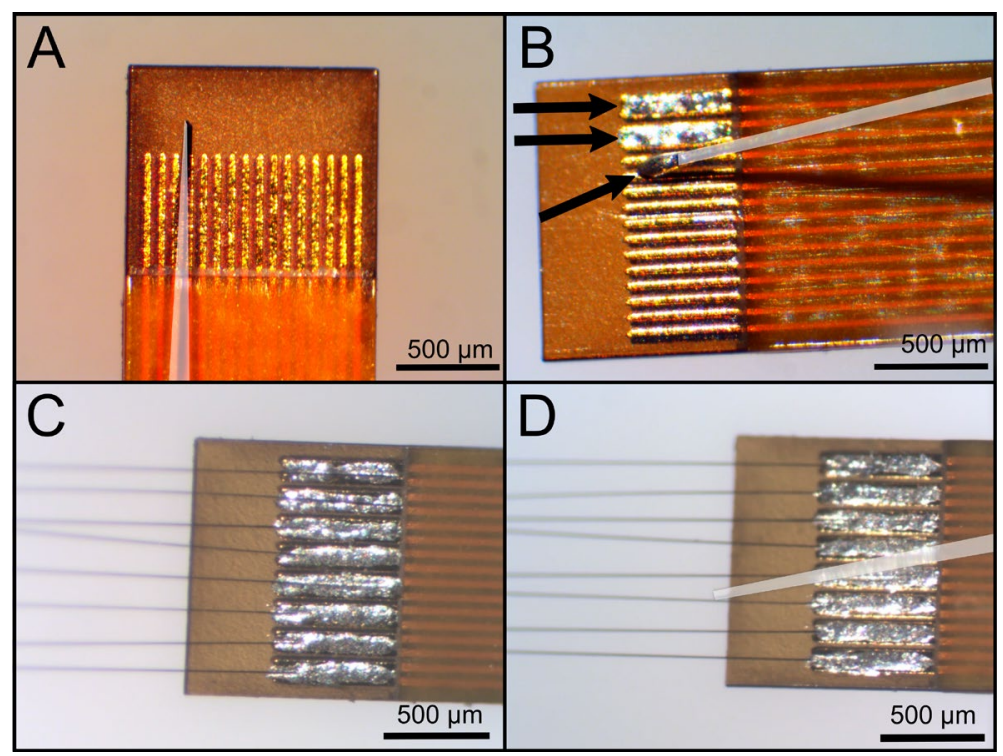

Figure 3: Applying silver epoxy and aligning carbon fibers in between the traces of the Flex Array. Capillaries have been highlighted with a white overlay. (A) The end of the capillary fits between the traces in order to get (B) clean silver epoxy (denoted with arrows at end of the capillary and within traces) deposition without spillover outside of trace pairs. (C) Carbon fibers are placed into the epoxy and then (D) straightened with a clean capillary.

Next, the traces were insulated with a small amount of UV epoxy (OG-143, Epoxy Technology, Billerica, MA) placed on the end of the board using a clean pulled glass capillary (Figure $4 \mathrm{~A}$ ). The amount of UV epoxy was enough to cover all traces and encapsulate all silver epoxy as this epoxy is meant to insulate the traces and fibers both from each other and from fluid interferences introduced in experiments. The probe was cured under a UV light (SpotCure-B6, Kinetic Instruments Inc, Bethel, CT) for a minimum of 2 minutes (Figure 4 B). The epoxy was checked by lightly tapping the surface with a clean pulled glass capillary to make sure it was fully cured (hard) before repeating on the other side; if not fully cured (sticky, soft), it was placed under the UV light for an additional 2 minutes. Once cured, fibers were cut to $1 \mathrm{~mm}$ lengths using stainless steel microsurgical scissors (15002-08, Fine Science Tools, Foster City, CA). When properly insulated, the board will have a small hard bubble on either side (Figure $4 \mathrm{~B}$ inset). 


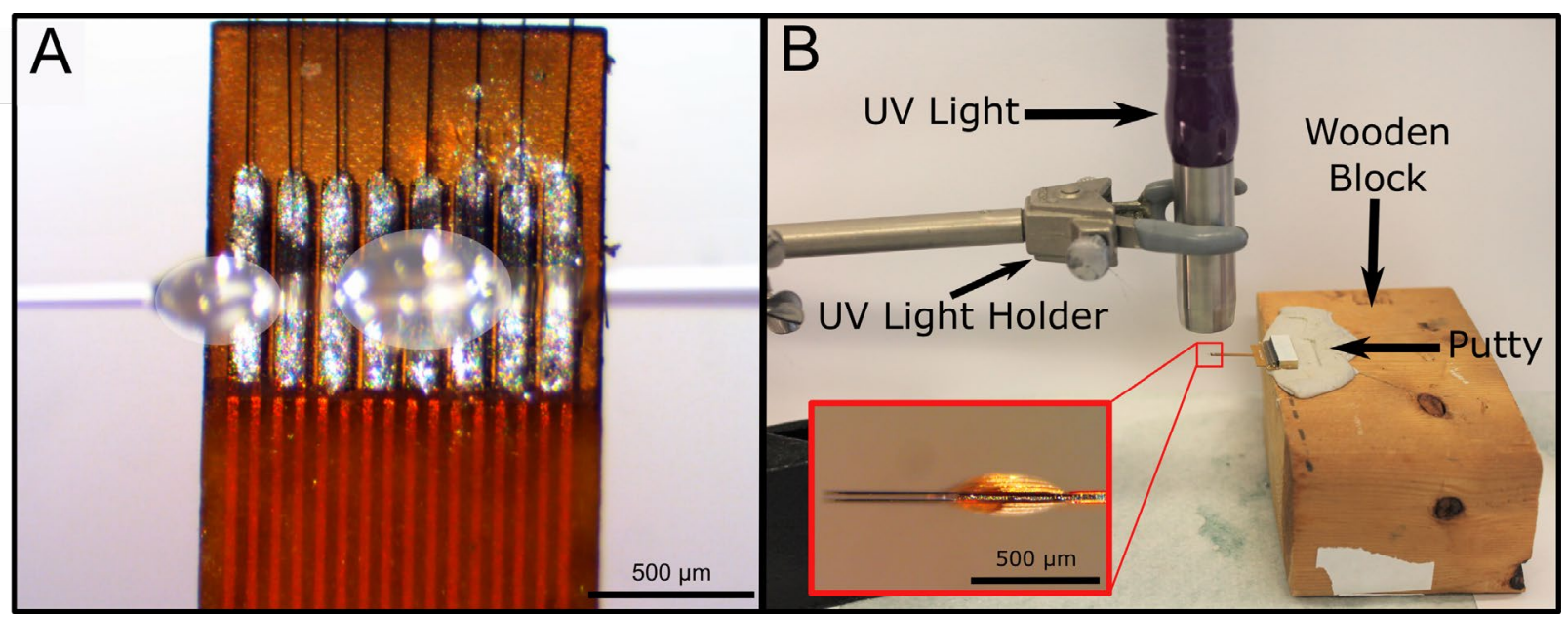

Figure 4: (A) UV epoxy is applied using a clean capillary and two drops of UV epoxy (marked with white overlays). UV epoxy is applied in droplets of 0.25-0.75 mm diameters until the UV epoxy forms a smooth bubble over the top of the traces. (B) Epoxy is cured under a UV light. The Flex Array is placed in putty on a wooden block for ease of movement and alignment underneath the UV light. The UV light is held with a holder about $1 \mathrm{~cm}$ above the end of the Flex Array. Inset (B) shows the side profile of a properly insulated Flex Array. The bubble on either side of the board is roughly $50 \mu \mathrm{m}$ in height.

\section{Checking Electrical Connections}

A $1 \mathrm{kHz}$ impedance scan was taken to confirm the fibers were electrically connected to the Omnetics connector and no shorts existed between fibers. Fibers were submerged $1 \mathrm{~mm}$ in $1 \times$ PBS (BP3994, Fisher, Waltham, MA) with an Ag|AgCl reference electrode (RE-5B, BASi, West Lafayette, MA) and a stainless-steel rod as the counter electrode. A PGSTAT12 Autolab (EcoChemie, Utrecht, Netherlands) and NOVA software provided by the vendor were used to take the measurements. Results were analyzed using custom MATLAB scripts (MathWorks, Natick, MA). Measurements were taken at multiple steps during the build process to verify connections. Typical impedance ranges varied depending on the build step (Table 3).

\begin{tabular}{c|c}
\hline BUILD STEP & EXPECTED 1KHZ IMPEDANCE $(\mathbf{k} \boldsymbol{\Omega})$ \\
\hline Bare Fiber & $150-300$ \\
Bare Fiber with UV & $400-500$ \\
Insulation & \\
Parylene-C & $>50,000$ \\
Insulated Fibers & $<15,000$ \\
Nd:YAG Laser Cut & $300-400$ \\
Blowtorched & $300-500$ \\
UV Laser Cut* & $<110$ \\
PEDOT:pTS Coated &
\end{tabular}

Table 3: Typical range of impedances after each build stage $(n=272){ }^{*} n=16$. PEDOT:pTS treated probes above $110 \mathrm{k} \Omega$ may still record signals, however all treated electrodes typically fall under this value. 
Once there was confidence in each build step, the number of impedance scans were reduced. Currently, they are performed only prior to parylene-c insulation and then as prescribed by the tip treatment procedure.

\section{Parylene-C Insulation}

The Flex Array's backend connector was masked using the mating connector (A79025-001) to prevent the internal pins of the Omnetics connector from being coated during the insulation process. A batch of arrays (8-12) were placed into a box with a raised, adhesive platform such that the connector end of the probe was resting on the platform and the majority of the board was overhanging the edge of the raised platform (Figure 5). We used inverted Fisher Tape super glued to a piece of foam as the raised platform.

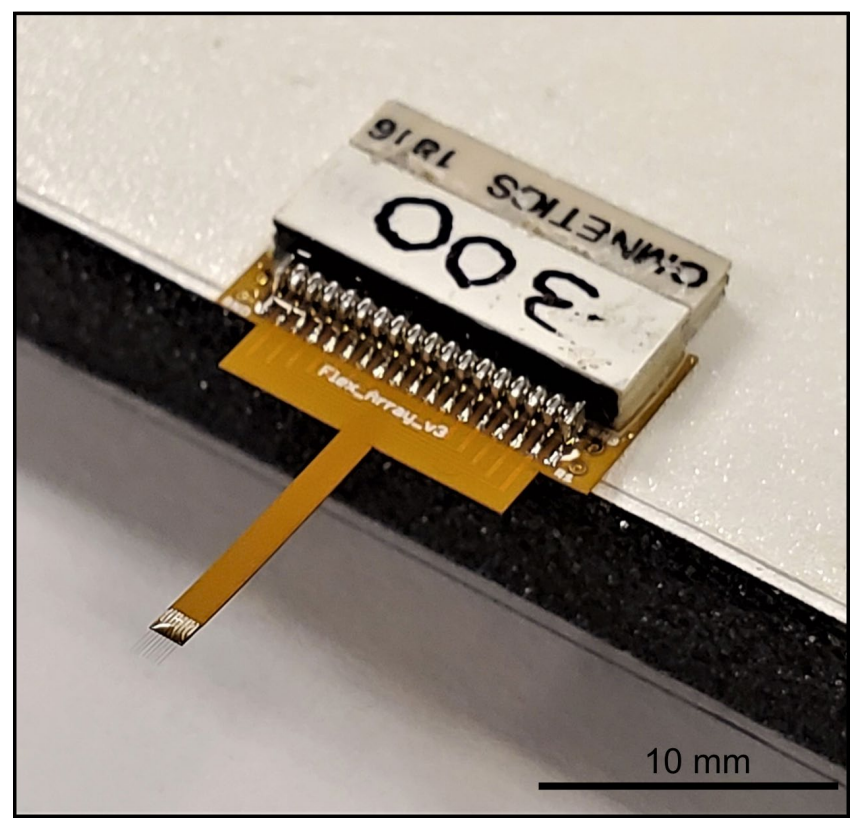

Figure 5: Flex Array prepared for parylene-c coating. The Flex Array is secured during the coating process to a raised foam platform with tape, adhesive side up.

Arrays were coated with a conformal layer of parylene-c (thickness $=800 \mathrm{~nm}$ ) using the Parylene-c Deposition System 2035 (Specialty Coatings Systems, Indianapolis, IN) located within the Lurie Nanofabrication Facility at the University of Michigan, following deposition rate guidelines of the machine. Many cleanrooms at research universities will have this deposition capability, which is easy for an individual to learn. A batch of 5 probes were sent to Specialty Coating Systems (Indianapolis, IN) to determine the viability of outsourcing this step to remove the only fabrication step that requires a cleanroom.

After parylene-c insulation, the backend masking was removed and the arrays were placed into a new box. A new box is required as the tape in the box that went through parylene-c deposition will be coated as well and unable to hold the arrays in place. The arrays were stored in a cool, dry, and dark place and considered shelf stable. An inventory of arrays was built up and used when needed for experiments.

\section{$\underline{\text { Tip Preparation Methods }}$}


One of three methods was used to re-expose carbon at the tip of the fiber: Nd:YAG laser cut, blowtorch, or ultraviolet (UV) laser. The fibers must be cut by one of these three methods as scissor cutting alone is not sufficient to reliably re-expose the tip of the carbon fiber [44]. Fibers under $500 \mu \mathrm{m}$ "self-insert" (require no additional or specialized insertion techniques) into the cortex [41], but for nerve or muscle a final length of $\leq 300 \mu \mathrm{m}$ with sharpened tips was required [45].

Nd:YAG Laser Cut

Fibers were first cut to $550 \mu \mathrm{m}$ with surgical scissors. A $532 \mathrm{~nm}$ Nd:YAG pulsed laser (LCS-1, New Wave Research, Fremont, CA: $5 \mathrm{~mJ} /$ pulse, $5 \mathrm{~ns}$ duration, $900 \mathrm{~mW}$ ) was used to further expose the carbon in conjunction with a Karl Suss probe station (LC3, SUSS MicroTec, Garching, Germany) for fiber alignment as shown previously [44]. The fibers were aligned inside of a $22 \times 50 \mu \mathrm{m}$ cutting window and the tips were cut off with 2-3 pulses resulting in a final length of $500 \mu \mathrm{m}$. The parylene-c ablated only slightly back $(<10 \mu \mathrm{m})$ from the tip after each cut [44].

\section{Blowtorch}

While Nd:YAG laser cut fibers reliably re-expose fiber tips, access to such a laser can be limiting. It also only provides blunt cylindrical electrode tips that have some difficulty inserting into muscle and nerve. Thus, a modified approach to previous sharpening methodology [40], [46] was taken using a butane blowtorch (Microtorch MT-51B, Master Appliance, Racine, WI). Fibers were cut to $300 \mu \mathrm{m}$ using surgical scissors. Using previously developed methods for nerve electrodes [47] an array was submerged in a dish of deionized water with the connector secured to the base of the dish with putty. The board was visually leveled and the water level was adjusted using a pipette and a pen camera (MS100, Teslong, Shenzhen, China) to ensure that the fibers were touching the surface of the water. A 3-5 mm flame from the blowtorch was run over the top of the fibers in a back-and-forth motion to sharpen the fibers (supplemental for video). The array was removed from the putty and inspected under a stereoscope for pointed tips. The process was repeated until points were able to be observed under a stereoscope.

\section{UV Laser Cut}

A UV laser can also be used to both cut and sharpen carbon fibers similarly to the blowtorch method. While the UV laser is currently unable to be used with Flex Arrays due to the board's small pitch size between fibers and rows of fibers, it does show promise with the larger pitch of the ZIF and Wide Board designs. This method is being developed to give a pathway to laser cutting with an easily obtainable UV laser to remove the access barrier that the Nd:YAG laser may provide. Thus, carbon fibers (2 mm length) were mounted on a ZIF and parylene-c insulated. A $1500 \mathrm{~mW}$ UV laser head (WER, Shanghai City, China) was affixed to three orthogonally configured motorized stages and then its focal point was moved across the fiber plane to cut the fibers to $500 \mu \mathrm{m}$ [48].

\section{PEDOT:pTS Coating}

For all tip cutting methods, an additional conductive layer must be added to the exposed carbon site to further reduce its impedance. In previous work, poly(3,4-ethylenedioxythiophene):sodium ptoulenesulfonate (PEDOT:PTS) has been used. A $50 \mathrm{~mL}$ solution of $0.01 \mathrm{M}$ 3,4-ethylenedioxythiophene (483028, Sigma-Aldrich, St. Louis, MO) and $0.1 \mathrm{M}$ sodium p-toluenesulfonate (152536, Sigma-Aldrich, St. Louis, MO) was stirred overnight, then refrigerated, and replaced every 30 days. This solution was stored in a light resistant container as it is light sensitive. 
Probe impedances were taken in 1x PBS solution with the same parameters used previously; "broken" (missing fiber) and "bad" (impedances $>1 \mathrm{M} \Omega$ ) channels were noted and not included in the PEDOT:pTS coating. Fibers with a good connection (typically 14-16 of the fibers) were electroplated with the PEDOT:PTS solution by applying 600 pA per fiber for 600 s using a PGSTAT12 Autolab. After electroplating, a final impedance measurement was taken and fibers with an impedance over $110 \mathrm{k} \Omega$ were designated "bad" in the probe's documentation.

\section{Finalizing the Probe}

The final step for finishing the probe was to solder ground and reference wires (Teflon Coated Silver Wire \#AGT05100, World Precision Instruments, Sarasota, FL) to the probes. As the ground and reference vias were coated in parylene-c, they were scraped clean with tweezers on the top and bottom of the board. Two $5 \mathrm{~cm}$ silver wires were de-insulated on either end ( $1 \mathrm{~cm}$ and $2 \mathrm{~mm}$ ). The $2 \mathrm{~mm}$ exposed portion of the wires were placed into the ground and reference vias and soldered into place. The excess wire was cut away from the backside solder joint (Figures $6 \mathrm{~A}$ and B). Probes were placed in a storage box with the reference and ground wires secured away from the electrode tips (Figure $6 \mathrm{C}$ ).

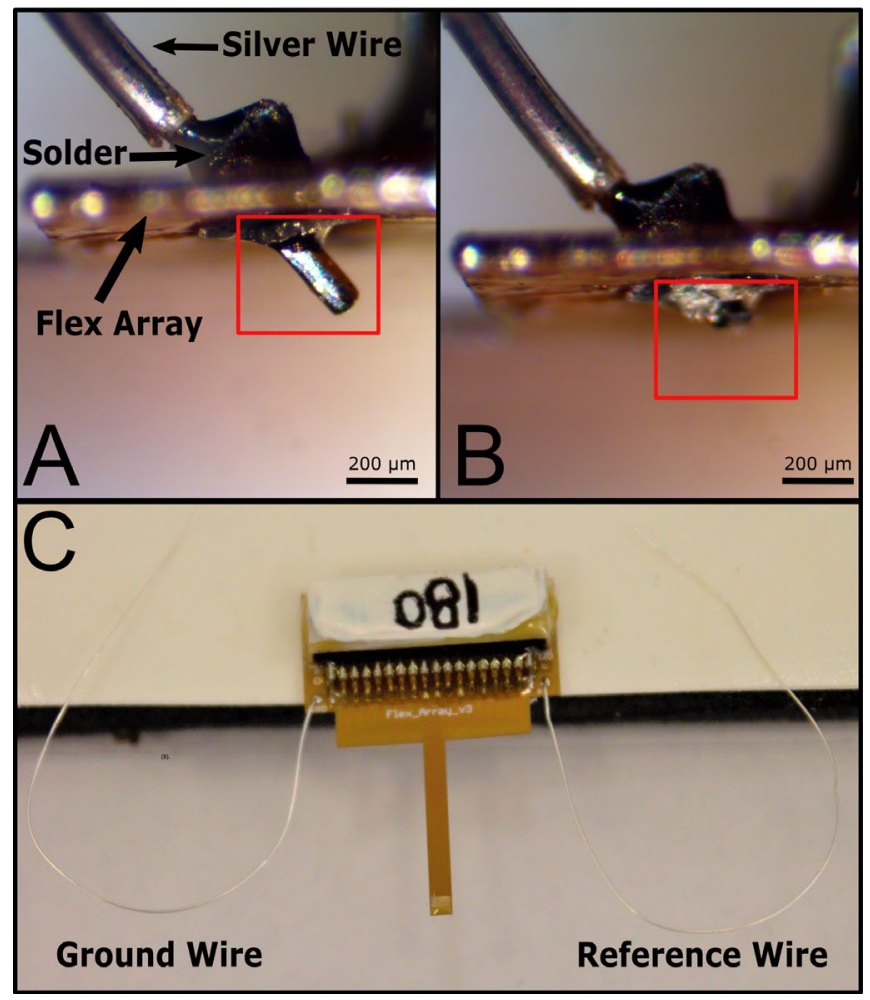

Figure 6: Ground and reference wires attached to the finalized Flex Array. Solder applied to each side of the via (A) and excess wire (red boxes) is (B) removed from the backside of the probe. Final Flex Array stored for future use.

\section{Surgery Protocol}

All animal procedures were approved by the University of Michigan Institutional Animal Care and Use Committee.

Surgical procedures for acute recordings followed Patel et al 2015 [41]. To summarize, an adult male Long Evans rat weighing approximately $300 \mathrm{~g}$ was anesthetized using a combination of ketamine 
(90 mg/kg) and xylazine (10 mg/kg). A bone screw (19010-00, Fine Science Tools, Foster City, CA) was used as the common reference and ground at the posterior edge of the skull. A $2.5 \mathrm{~mm}$ by $2.5 \mathrm{~mm}$ craniotomy was made over the right hemisphere's motor cortex. After dura resection, a ZIF array with 4 UV laser treated fibers was inserted to a depth of $1.2 \mathrm{~mm}$. All ePhys data was collected using a ZC16 headstage, RA16PA pre-amplifier, and RX5 Pentusa base station (Tucker-Davis Technologies, Alachua, $\mathrm{FL}$ ). The pre-amplifier high-pass filtered at $2.2 \mathrm{~Hz}$, anti-alias filtered at $7.5 \mathrm{kHz}$, and sampled at $25 \mathrm{kHz}$. The recording session was 10 minutes long.

\section{Spike Sorting}

Offline Sorter (OFS, Plexon, Dallas, TX) software was used to spike sort the data following the methods outlined in [49]. Channels were high-pass filtered ( $250 \mathrm{~Hz}$ corner, $4^{\text {th }}$ order Butterworth) and waveforms were detected at $-3.5^{*} \mathrm{RMS}$ threshold. Cluster centers were identified in principle component states using a K-means sorting method. Obvious noise clusters were eliminated from the data set. A Gaussian model was used to cluster the remaining clusters. Spikes with similar characteristics were combined and averaged over the cluster. Carbon fiber electrodes with discernible units were deemed viable. A minimum of 10 waveforms were required for a unit to be included in the data.

\section{$\underline{\text { SEM Imaging }}$}

An FEI Nova 200 Nanolab Focused Ion Beam Workstation and Scanning Electron Microscope (FEI, Hillsboro, OR) was used for SEM imaging of Nd:YAG laser and blowtorch prepared fibers. Prior to imaging, samples were gold sputter coated with an SPI-Module Sputter Coater (SPI Supplies, West Chester, PA). Images of UV laser prepared fibers were obtained with the JEOL InTouchScope Scanning Electrode Microscope (JSM-IT500HR, JEOL, Tokyo, Japan). 


\section{Results}

\section{Tip Validation: SEM Images}

Previous work [44] showed that scissor cutting resulted in unreliable impedances as parylene-c folded across the recording site. Scissor cutting is used here only to cut fibers to a desired length before processing with an additional finish cutting method. SEM images of the tips were used to determine exposed carbon length and tip geometry (Figure 7).

Scissor and Nd:YAG laser cut fibers were previously reviewed [35], [44]. Scissor cut fibers (Figure 7 A) have inconsistent tip geometries with parylene-c folding over the end when cut [44]. The Nd:YAG laser cut fibers remain consistent in recording site area, shape, and impedance (Figure 7 B). Blowtorched fibers [47] lead to the highest electrode size and shape variability, but also resulted in a sharpened tip allowing for insertion into tough tissue and on average $140 \mu \mathrm{m}$ of carbon was re-exposed with a smooth transition area between the carbon and parylene-c insulation. UV laser cut fibers were similar to with blowtorched fibers, showing $120 \mu \mathrm{m}$ of carbon exposed from the tip. Impedances indicated that either the UV laser or blowtorch tip cutting methods are suitable for ePhys and are viable solutions for labs without access to an Nd:YAG laser.

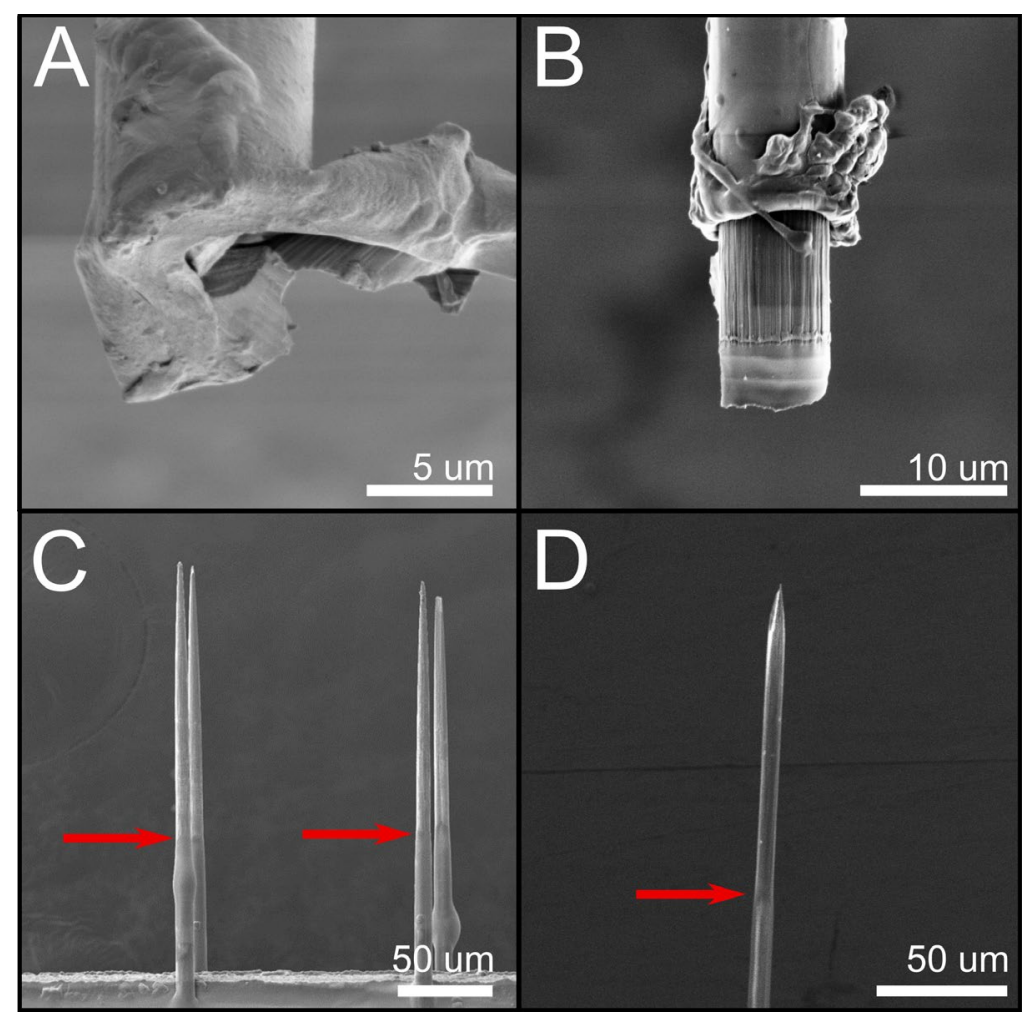

Figure 7: SEM images of fibers with different tip cutting techniques. (A) Scissor cut fiber with very little exposed carbon. (B) Nd:YAG laser cut. (C) Blowtorched fiber with 140 $\mu \mathrm{m}$ of carbon exposed from the tip. (D) UV laser cut fibers with $\sim 120 \mu \mathrm{m}$ of carbon exposed from the tip. Red arrows indicate the transition area between parylene and bare carbon fiber.

\section{Tip Validation: Electrical Recording}

Figure 8 shows resulting impedances from each preparation method using Flex Arrays. The resultant values are within an appropriate range for ePhys recording. Nd:YAG laser cut fibers resulted in 
the smallest surface area but the highest impedances, even with the PEDOT:pTS coating (bare carbon: $4138 \pm 1438 \mathrm{k} \Omega$; with PEDOT:pTS: $27 \pm 18 \mathrm{k} \Omega ; \mathrm{n}=262$ ). This is followed by the inverse relationship in blowtorched (bare carbon: $308 \pm 121 \mathrm{k} \Omega$; with PEDOT:pTS: $16 \pm 13 \mathrm{k} \Omega ; \mathrm{n}=262$ ) and UV Laser cut (bare carbon: $468 \pm 342 \mathrm{k} \Omega$; with PEDOT:pTS: $27 \pm 8 \mathrm{k} \Omega ; \mathrm{n}=7$ ) fibers that have a large surface area and low impedances. However, in all cases, the PEDOT:pTS coated fibers do fall under the $110 \mathrm{k} \Omega$ threshold that was set previously to indicate a good, low impedance electrode.

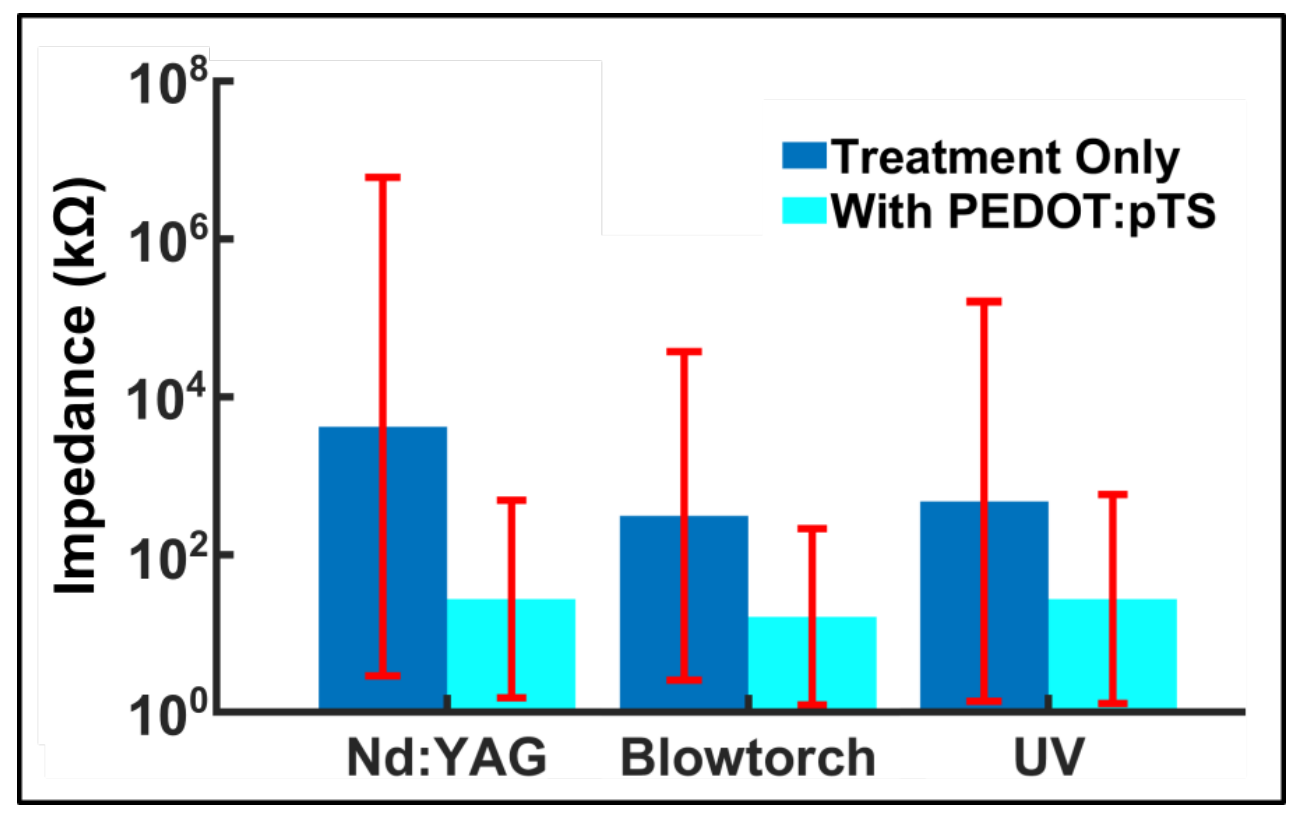

Figure 8: Impedance (mean \pm standard deviation) differences between only applying the treatment (bare carbon exposed) and with the addition of PEDOT:pTS. In all cases the addition of PEDOT:pTS decreases the impedance by an order of magnitude.

Acute ePhys recordings were taken from a Long Evans rat acutely implanted with a ZIF array with UV laser cut and PEDOT:pTS treated fibers to demonstrate the viability of this method. EPhys has previously been tested and proven with scissor cut [44], Nd:YAG [35], and blowtorch treated fibers [45], [49]. Acute recordings from four UV laser treatment fibers that were simultaneously implanted in rat motor cortex $(n=1)$ are presented in Figure 9 . Three units were found across all fibers suggesting that the treatment of the fibers with the inexpensive UV laser is similar to other cutting methods that enable the carbon fiber to record neural units, as would be expected by the SEMs and impedances. 


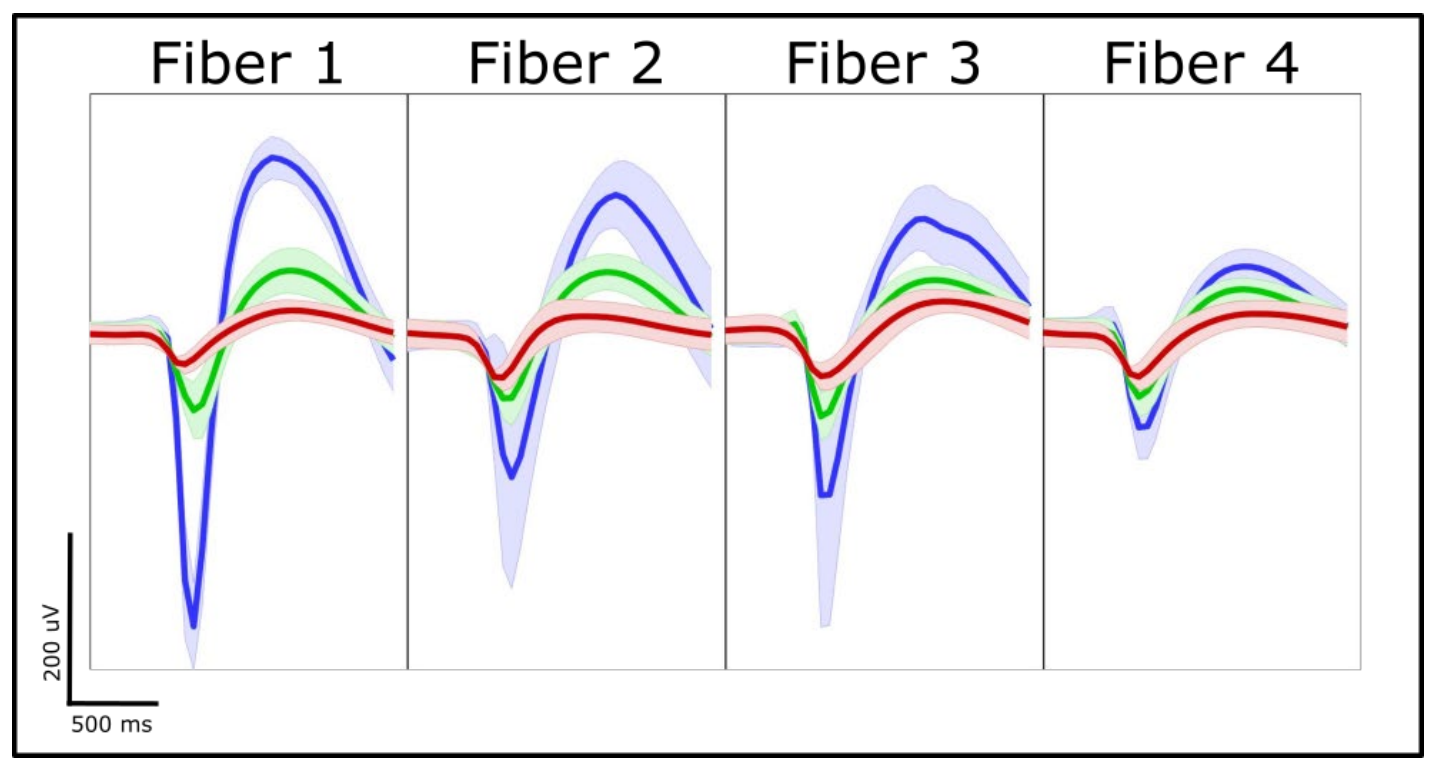

Figure 9: Acute electrophysiological spiking data from four UV laser cut electrodes.

While carbon fiber arrays are easily built and modified to suit the user's needs, it should be noted that additional validation is necessary for some builds (Table 4) while others are less suitable for certain end tasks.

PREPARATION METHOD

Nd:YAG

Blowtorch

UV Laser
WIDE BOARD Impedance, SEM, acute ePhys Impedance, SEM, acute ePhys

Not yet validated
ZIF Impedance, SEM, acute/chronic ePhys Impedance, SEM, acute/chronic ePhys Impedance, SEM, acute/chronic ePhys
FLEX ARRAY

Impedance, SEM, acute/chronic ePhys Impedance, SEM, acute/chronic ePhys

Not Viable

Table 4: Validated uses of each board with the cutting methods described. All cutting methods included electrodeposition of PEDOT:pTS. 'Not Viable' indicates that a form factor of the design prevents this tip treatment from being tested at this time (i.e. fiber pitch).

\section{Commercial Parylene-c}

Commercially coated arrays were determined to have a parylene-c thickness of $710 \mathrm{~nm}$ by the vendor, well within the target range of insulation. The arrays were prepared for ePhys recordings using the blowtorch tip preparation. Impedances were taken after preparation of the tips and compared to existing data. A blowtorched and PEDOT:pTS coated probe had an average of $14.5 \pm 1.3 \mathrm{k} \Omega$ impedance across 16 fibers. SEM images were taken of the tip and shank to compare parylene-c deposition (Figures $10 \mathrm{~A}$ and $\mathrm{B}$, respectively). These results show the use of a commercial vendor did not change expected 
impedance values, suggesting that this will be an equally viable substitution to deposition at the university cleanroom.

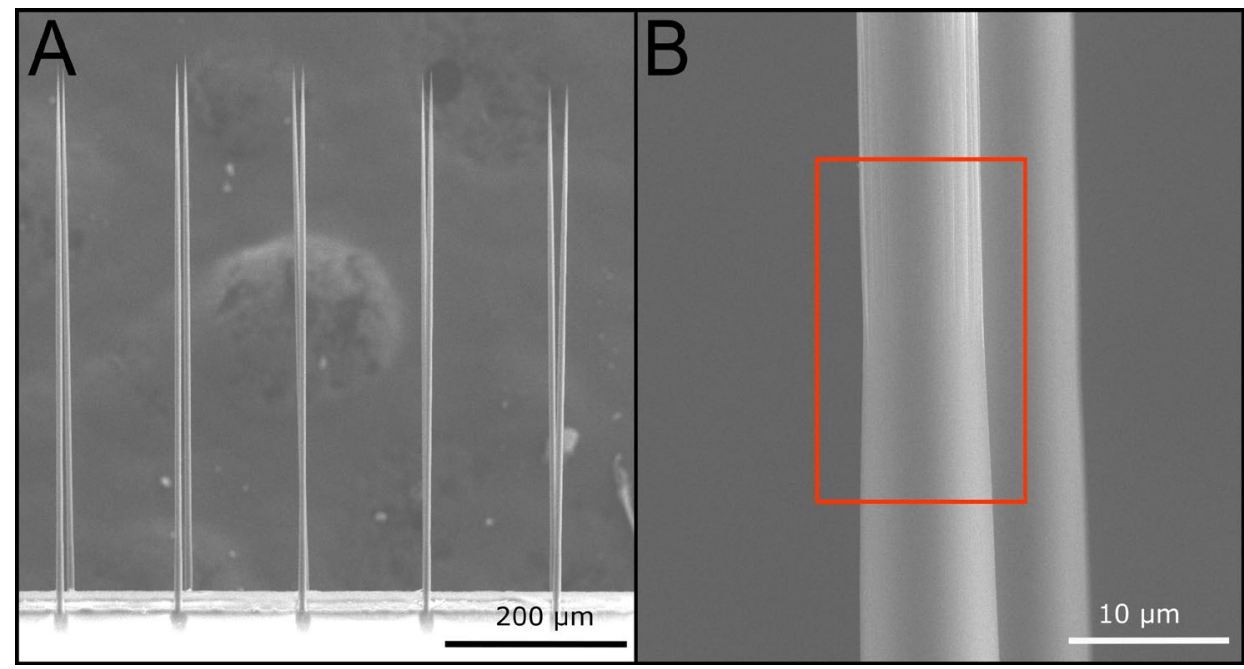

Figure 10: Commercial parylene-c coated arrays. (A) The sharpened array shows uniform sharpening across all fibers indicating that there are no drawbacks to commercial coating. (B) The transition (red box) between bare carbon fiber and parylene-c after blowtorching shows no discernable difference between arrays coated in a cleanroom facility.

\section{Device Cost Analysis}

Provided all tools and bulk materials (epoxies, solder, etc.) are accessible to the researcher, a parylene-c user fee of $\$ 41$, and a batch of 8 probes, the total materials cost is $\$ 1168$ ( $\$ 146$ per probe). Personnel effort (Table 5 ) is around 25 hours for the batch. If using a substituted fabrication step, the cost of the probes will vary based on commercial parylene-c coating cost ( $\$ 500-800$ quoted). The time for build steps (Table 5) is grouped together for all instances of a repeated tasks for simplicity. Build times for designs with a larger pitch (Wide Board and ZIF) are dramatically reduced as the manually intensive steps (e.g. carbon fiber placement) are easier and faster to complete.

ACTIVITY

\begin{tabular}{c}
\hline All Soldering \\
Insulating Omnetics \\
Populating Carbon Fibers \\
Insulating Traces with UV Epoxy \\
Parylene-C Deposition \\
Nd:YAG Laser Cutting \\
Blowtorching \\
UV LASER CUTTING \\
All Impedance Testing \\
PEDOT:pTS Deposition \\
Recipe Used \\
Nd:YAG Laser Cut
\end{tabular}

TIME FOR 8 DEVICES (HRS)

5
1
10
0.5
1.5
1
1
1.5
4.5
1.5
Total Hours
25


bioRxiv preprint doi: https://doi.org/10.1101/2021.03.22.436422; this version posted March 22, 2021. The copyright holder for this preprint (which was not certified by peer review) is the author/funder, who has granted bioRxiv a license to display the preprint in perpetuity. It is made available under aCC-BY-NC-ND 4.0 International license.

\section{Blowtorch \\ UV Laser Cut}

25.5

Table 5: Time required for each step of a fabrication process. Soldering of the Omnetics and ground and reference wires have been combined here to simplify the activity list. 


\section{Discussion}

\section{Troubleshooting Build Issues}

Silver epoxy deposition tends to fail for several reasons: the width of the capillary is too wide to fit between traces, the width of the capillary is too thin to pick up and deposit epoxy, or an excess of epoxy is on the capillary. The first two problems can be solved by cutting a new capillary that is a more appropriate size; the latter by dipping the capillary into the epoxy with a lighter hand or removing a portion of the epoxy blob by gently dabbing the capillary onto a spare nitrile glove.

Deciding how to prepare the electrode is often a difficult decision for many users. However, determining what is needed for the experiment will help illuminate the decision. For acute surgeries, blunt tips can be used if the site size of the electrode is important, however they will only insert into softer tissue (brain) and only at sub-500 $\mu \mathrm{m}$ target depths. Going into deeper brain structures is possible using a glass cannula[50], but this can cause scarring and associated unreliability in ePhys recordings. Fibers must be less than $300 \mu \mathrm{m}$ when sharpened to be able to penetrate the nerve tissue as the shorter length provides a stiffer backbone for insertion. Sharpened fibers have also recently been observed to penetrate to $1 \mathrm{~mm}$ depths in the brain [49].

While the arrays discussed in this paper are an excellent starting point for many labs, newer probes using carbon fibers have also been developed to chronically target deeper areas. The Chestek lab high density carbon fiber array provide users the ability to insert 16 carbon fibers to depths up to $9 \mathrm{~mm}$ with minimal scaring [50]. The Maharbiz lab has also developed a higher channel probe that was inserted to depths of 2.5-3 mm [51]. The Cox lab has also developed a carbon fiber bundle probe $\sim 1 \mathrm{~mm}$ in length [39]. These probe geometries will be of greater use to labs doing chronic work in deeper brain structures.

Parylene-C Accessibility

Parylene- $\mathrm{C}$ is a method of conformal coating at room temperature that has been used as a biocompatible insulator in many implanted devices. The technique requires a specialized tool in a cleanroom and takes about an hour to learn. A cursory survey of institutions that have previously requested carbon fiber arrays from our group was conducted to determine parylene-c deposition accessibility. We found that out of 17 institutes, $41 \%$ had access to parylene-c coating systems on their campus. For universities without access to a parylene-c coating system, commercial coating services are a viable alternative as we have demonstrated. Alternatively, outsourcing to a nearby university cleanroom may also be of interest to labs with no direct access to a parylene-c deposition system. To reduce the cost per device, we advise sending out larger batches of arrays as commercial systems are often able to accommodate larger samples.

\section{Optimizing Tip Preparations}

Additional tip preparations need to be investigated for these fibers as the current tip preparations do require the end user to choose between penetrating ability and a small recording site. While the $\mathrm{Nd}$ :YAG laser cut fibers provide a small site size [44], the ability to penetrate stiffer tissue (muscle, nerve) is almost non-existent and access to a laser setup capable of this cutting technique can be difficult and expensive. While blowtorching allows for a quick and economical way to get sharpened tips that can penetrate many tissues [45], the tip geometry is large and may be inconsistent from fiber to fiber [47]. UV 
laser cutting falls to the same issues with the added obstacle of having to engineer a way to align the laser with fibers and current restrictions on using this method with the Flex Array due to the pitch of the fibers being smaller than the laser's focal point diameter. Previous work showed fabrication of small, sharpened fibers via etching [52], [53]. This approach could result in a small, reliable electrode geometry and would preserve the sharpened tip necessary for penetrating nerve and muscle.

Our current tip coating, PEDOT:pTS, may also need to be replaced as it tends to degrade overtime, which is an undesirable trait for a chronic probe [35], [44], [54]. A lack of PEDOT:pTS longevity, leads to higher impedances and therefore lower signal quality in part due to increased background noise. To increase longevity in our fiber tips, investigation into the feasibility of platinum-iridium coatings is being conducted. Platinum-iridium would allow for a greater surface area [44], [55] concentrated on the tip of the electrode, keeping a low impedance and [55]-[57] allow for longer, chronic stability [55], [57].

Other coatings such as PEDOT/graphene oxide [58] and gold [59] have been utilized to lower carbon fiber electrode impedances, though these coatings are typically used for chemical sensing probes rather than for ePhys recordings. Due to the inherent properties of carbon fibers [60], the carbon fiber array presented here can be converted from a probe optimized for ePhys to a chemical sensing device with a simple change of tip preparation [50].

\section{Further Experimental Options}

The carbon fibers arrays in this paper have also been successfully fabricated using the methods presented here and utilized to create dopamine sensing arrays. This is accomplished by ablating the parylene-c away from the tip of the probe using the Nd:YAG laser, at a lower power setting, and exposing $50 \mu \mathrm{m}$ of fiber [50]. Using custom hardware and software, simultaneous high density channel recording has recently been achieved [50]. The probe's ability to be customized for ePhys or dopaminergic recordings offers yet another layer of adaptability to our carbon fiber array. Coupled with the new coating techniques previously mentioned, this opens up a variety of possible probe variants that could be made and modified, quickly and inexpensively.

\section{Automated Carbon Fiber Placement}

The manual population of the carbon fibers is labor intensive and has a steep learning curve. Many new users struggle with the fibers breaking during handling, flying away due to static forces, and seeing the fibers when not under the stereoscope. While these issues can be addressed with some modifications to the work area, the process is still intensive and slow. Automated carbon fiber placement of an array has been achieved in a different probe configuration [42] in the Maharbiz lab. This technology allows for carbon fiber to be inserted and aligned into a probe with the use of a camera, motorized stages, custom guides, and a custom computer algorithm. Modifying this set up would allow for new users to avoid the learning curve of handling small (2-4 mm long) fibers and allow them to manually feed a longer fiber ( $>1$ $\mathrm{cm}$ ) into a machine that would then place and align the fibers. If this technology were implemented, probes would be able to be made faster and more accurately with less training required. However, using the approaches discussed in this work, we believe that virtually any lab with experience working under a stereoscope should be able to fabricate their own fully functional carbon fiber electrode arrays.

\section{Acknowledgment}


This work was financially supported by National Institutes of Health National Institutes of Neurological Disorders and Stroke (UF1NS107659 and UF1NS115817) and the National Science Foundation (1707316). The authors acknowledge financial support from the University of Michigan College of Engineering and technical support from the Michigan Center for Materials Characterization and the Van Vlack Undergraduate Laboratory. The authors thank Dr Khalil Najafi for use of his Nd:YAG laser and the Laurie Nanofabrication Facility for use of their parylene-c deposition machine.

\section{Citations}

[1] K. M. Szostak, L. Grand, and T. G. Constandinou, "Neural interfaces for intracortical recording: Requirements, fabrication methods, and characteristics," Frontiers in Neuroscience, vol. 11, no. DEC. Frontiers Media S.A., 07-Dec-2017, doi: 10.3389/fnins.2017.00665.

[2] J. D. Simeral, S. Kim, M. J. Black, and J. P. Donoghue, "Neural control of cursor trajectory and click by a human with tetraplegia 1000 days after implant of an intracortical microelectrode array," vol. 025027, 2011, doi: 10.1088/1741-2560/8/2/025027.

[3] L. R. Hochberg et al., "Neuronal ensemble control of prosthetic devices by a human with tetraplegia," Nature, vol. 442, no. 7099, pp. 164-171, Jul. 2006, doi: 10.1038/nature04970.

[4] L. Yang, K. Lee, J. Villagracia, and S. C. Masmanidis, "Open source silicon microprobes for high throughput neural recording," J. Neural Eng., vol. 17, no. 1, 2020, doi: 10.1088/17412552/ab581a.

[5] L. Luan et al., "Ultraflexible nanoelectronic probes form reliable , glial scar - free neural integration," no. February, pp. 1-10, 2017, doi: 10.1126/sciadv.1601966.

[6] Z. Jeff et al., "Ultrasoft microwire neural electrodes improve chronic tissue integration," Acta Biomater., vol. 53, pp. 46-58, 2017, doi: 10.1016/j.actbio.2017.02.010.

[7] M. A. Moffitt and C. C. McIntyre, "Model-based analysis of cortical recording with silicon microelectrodes," Clin. Neurophysiol., vol. 116, no. 9, pp. 2240-2250, Sep. 2005, doi: 10.1016/j.clinph.2005.05.018.

[8] E. M. Maynard, C. T. Nordhausen, and R. A. Normann, "The Utah Intracortical Electrode Array : a recording structure for potential brain-computer interfaces," vol. 102, pp. 228-239, 1997.

[9] H. A. C. Wark et al., "A new high-density (25 electrodes $/ \mathrm{mm} 2$ ) penetrating microelectrode array for recording and stimulating sub-millimeter neuroanatomical structures," J. Neural Eng., vol. 10, no. 4, 2013, doi: 10.1088/1741-2560/10/4/045003.

[10] A. Sharma et al., "Long term in vitro functional stability and recording longevity of fully integrated wireless neural interfaces based on the Utah Slant Electrode Array," vol. 045004, 2011, doi: 10.1088/1741-2560/8/4/045004.

[11] C. A. Chestek et al., "Long-term stability of neural prosthetic control signals from silicon cortical arrays in rhesus macaque motor cortex," J. Neural Eng., vol. 045005, no. 4, Aug. 2011, doi: 10.1088/1741-2560/8/4/045005.

[12] J. C. Barrese et al., "Failure mode analysis of silicon-based intracortical microelectrode arrays in non-human primates," J. Neural Eng., vol. 066014, no. 6, 2013, doi: 10.1088/1741- 


\section{0/10/6/066014.}

[13] X. Xie et al., "Long-term reliability of Al 203 and Parylene $\mathrm{C}$ bilayer encapsulated Utah electrode array based neural interfaces for chronic implantation," vol. 026016, doi: 10.1088/17412560/11/2/026016.

[14] D. R. Kipke, R. J. Vetter, J. C. Williams, and J. F. Hetke, "Silicon-Substrate Intracortical Microelectrode Arrays for Long-Term Recording of Neuronal Spike Activity in Cerebral Cortex," IEEE Trans. Neural Syst. Rehabil. Eng., vol. 11, no. 2, pp. 151-155, Jun. 2003, doi: 10.1109/TNSRE.2003.814443.

[15] A. Bragin, J. Hetke, C. L. Wilson, D. J. Anderson, J. Engel, and G. Buzsáki, "Multiple site siliconbased probes for chronic recordings in freely moving rats: Implantation, recording and histological verification," J. Neurosci. Methods, vol. 98, no. 1, pp. 77-82, 2000, doi: 10.1016/S0165-0270(00)00193-X.

[16] J. J. Jun et al., "Fully integrated silicon probes for high-density recording of neural activity," Nature, vol. 551, no. 7679, 2017, doi: 10.1038/nature24636.

[17] A. S. Caravaca et al., "A novel flexible cuff-like microelectrode for dual purpose, acute and chronic electrical interfacing with the mouse cervical vagus nerve," J. Memb. Sci., pp. 0-27, 2017, doi: 10.1016/j.memsci.2007.08.033.

[18] K. D. Wise, D. J. Anderson, J. F. Hetke, D. R. Kipke, and K. Najafi, "Wireless implantable microsystems: High-density electronic interfaces to the nervous system," in Proceedings of the IEEE, 2004, vol. 92, no. 1, pp. 76-97, doi: 10.1109/JPROC.2003.820544.

[19] M. P. Ward, P. Rajdev, C. Ellison, and P. P. Irazoqui, "Toward a comparison of microelectrodes for acute and chronic recordings," Brain Res., vol. 1282, pp. 183-200, Jul. 2009, doi: 10.1016/j.brainres.2009.05.052.

[20] R. Biran, D. C. Martin, and P. A. Tresco, "Neuronal cell loss accompanies the brain tissue response to chronically implanted silicon microelectrode arrays," vol. 195, no. 1, pp. 115-126, Sep. 2005, doi: 10.1016/j.expneurol.2005.04.020.

[21] M. B. Christensen, S. M. Pearce, N. M. Ledbetter, D. J. Warren, G. A. Clark, and P. A. Tresco, "The foreign body response to the Utah Slant Electrode Array in the cat sciatic nerve," Acta Biomater., vol. 10, no. 11, 2014, doi: 10.1016/j.actbio.2014.07.010.

[22] R. Biran, D. C. Martin, and P. A. Tresco, "The brain tissue response to implanted silicon microelectrode arrays is increased when the device is tethered to the skull," J. Biomed. Mater. Res. - Part A, vol. 82, no. 1, pp. 169-178, 2007, doi: 10.1002/jbm.a.31138.

[23] T. D. Y. Kozai et al., "Mechanical failure modes of chronically implanted planar silicon-based neural probes for laminar recording," Biomaterials, vol. 37, pp. 25-39, Jan. 2015, doi: 10.1016/j.biomaterials.2014.10.040.

[24] K. Scholten and E. Meng, "Materials for microfabricated implantable devices: A review," Lab Chip, vol. 15, no. 22, pp. 4256-4272, 2015, doi: 10.1039/c5lc00809c.

[25] R. Caldwell, M. G. Street, R. Sharma, P. Takmakov, B. Baker, and L. Rieth, "Characterization of Parylene-C degradation mechanisms : In vitro reactive accelerated aging model compared to multiyear in vivo implantation," Biomaterials, vol. 232, no. January 2019, p. 119731, 2020, doi: 
10.1016/j.biomaterials.2019.119731.

[26] Z. Zhao, X. Li, F. He, X. Wei, S. Lin, and C. Xie, "Parallel, minimally-invasive implantation of ultraflexible neural electrode arrays," J. Neural Eng., 2019, doi: 10.1088/1741-2552/ab05b6.

[27] L. Luan et al., "Ultraflexible nanoelectronic probes form reliable, glial scar-free neural integration."

[28] F. Deku, Y. Cohen, A. Joshi-Imre, A. Kanneganti, T. Gardner, and S. Cogan, "Amorphous silicon carbide ultramicroelectrode arrays for neural stimulation and recording," J. Neural Eng., pp. 020, 2018, doi: https://doi.org/10.1088/1361-6528/aa8b39.

[29] S. Vasudevan, K. Patel, and C. Welle, "Rodent model for assessing the long term safety and performance of peripheral nerve recording electrodes," J. Neural Eng., vol. 14, no. 1, 2017, doi: 10.1088/1741-2552/14/1/016008.

[30] A. Prasad et al., "Comprehensive characterization and failure modes of tungsten microwire," vol. 056015, 2012, doi: 10.1088/1741-2560/9/5/056015.

[31] M. Sawan, "A Review of Microelectrode Array Technologies : Design and Implementation Challenges," 2013 2nd Int. Conf. Adv. Biomed. Eng., pp. 38-41, 2013, doi:

10.1109/ICABME.2013.6648841.

[32] B. D. Winslow and P. A. Tresco, "Biomaterials Quantitative analysis of the tissue response to chronically implanted microwire electrodes in rat cortex," Biomaterials, vol. 31, no. 7, pp. 15581567, 2010, doi: 10.1016/j.biomaterials.2009.11.049.

[33] W. M. Tsang and M. Je, "Flexible electrode for implantable neural devices," in Neural Computation, Neural Devices, and Neural Prosthesis, Springer New York, 2014, pp. 121-156.

[34] A. Obaid et al., "Massively Parallel Microwire Arrays Integrated with CMOS chips for Neural Recording," 2019, doi: 10.1101/573295.

[35] P. R. Patel et al., "Chronic in vivo stability assessment of carbon fiber microelectrode arrays," J. Neural Eng., vol. G, no. 6, p. 66002, 2016, doi: 10.1088/1741-2560/13/6/066002.

[36] T. D. Yoshida Kozai et al., "Ultrasmall implantable composite microelectrodes with bioactive surfaces for chronic neural interfaces," Nat. Mater., vol. 11, no. 12, pp. 1065-1073, Dec. 2012, doi: $10.1038 / \mathrm{nmat} 3468$.

[37] N. Saito et al., "Application of carbon fibers to biomaterials: A new era of nano-level control of carbon fibers after 30-years of development," Chem. Soc. Rev., vol. 40, no. 7, pp. 3824-3834, 2011, doi: 10.1039/c0cs00120a.

[38] H. N. Schwerdt et al., "Lab on a Chip from multiple brain sites †," Lab Chip, vol. 17, pp. 11041115, 2017, doi: 10.1039/C6LC01398H.

[39] G. Guitchounts and D. Cox, "64-Channel Carbon Fiber Electrode Arrays for Chronic Electrophysiology," 2019, doi: 10.1101/697409.

[40] G. Guitchounts, J. E. Markowitz, W. A. Liberti, and T. J. Gardner, "A carbon-fiber electrode array for long-term neural recording," J. Neural Eng., vol. 046016, no. 4, Aug. 2013, doi: 10.1088/17412560/10/4/046016. 
[41] P. R. Patel et al., "Insertion of linear $8.4 \mu \mathrm{m}$ diameter 16 channel carbon fiber electrode arrays for single unit recordings," J. Neural Eng., vol. 12, no. 4, p. 46009, 2015, doi: 10.1088/17412560/12/4/046009.

[42] T. L. Massey et al., "Open-Source Automated System for Assembling a High-Density Microwire Neural Recording Array."

[43] H. N. Schwerdt et al., "Subcellular probes for neurochemical recording from multiple brain sites," Lab Chip, 2017, doi: 10.1039/C6LC01398H.

[44] E. J. Welle, P. R. Patel, J. E. Woods, A. Petrossians, and E. Valle, "Ultra-small carbon fiber electrode recording site optimization and improved in vivo chronic recording yield Ultra-small carbon fiber electrode recording site optimization and improved in vivo chronic recording yield," 2020.

[45] A. A. Jiman et al., "Multi-channel intraneural vagus nerve recordings with a novel high-density carbon fiber microelectrode array," 2020.

[46] W. F. Gillis et al., "Carbon fiber on polyimide ultra-microelectrodes," J. Neural Eng., vol. 15, no. 1, Feb. 2018, doi: 10.1088/1741-2552/aa8c88.

[47] E. J. Welle et al., "Fabrication and characterization of a carbon fiber peripheral nerve electrode appropriate for chronic recording," 2019.

[48] T. Dong, L. Chen, and A. Shih, "Laser Sharpening of Carbon Fiber Microelectrode Arrays for Brain Recording," J. Micro Nano-Manufacturing, 2021, doi: 10.1115/1.4049780.

[49] E. J. Welle et al., "Sharpened and mechanically robust carbon fiber electrode arrays for neural interfacing," bioRxiv, pp. 1-18, 2021.

[50] P. R. Patel et al., "High density carbon fiber arrays for chronic electrophysiology, fast scan cyclic voltammetry, and correlative anatomy," J. Neural Eng., vol. 17, no. 5, Oct. 2020, doi: 10.1088/1741-2552/abb1f6.

[51] T. L. Massey, S. R. Santacruz, J. F. Hou, K. S. J. Pister, J. M. Carmena, and M. M. Maharbiz, “A highdensity carbon fiber neural recording array technology," J. Neural Eng., vol. 16, no. 016024, 2019.

[52] H. Khani, D. O. Wipf, J. E. Soc, and D. O. Wipf, "Fabrication of Tip-Protected Polymer-Coated Carbon-Fiber Ultramicroelectrodes and pH Ultramicroelectrodes Ultramicroelectrodes and $\mathrm{pH}$ Ultramicroelectrodes," 2019, doi: 10.1149/2.0941908jes.

[53] C. Ultramicroelectrodes, E. E. M. El-giar, and D. O. Wipf, "Preparation of Tip-Protected Poly ( oxyphenylene ) Coated," no. lii, pp. 2281-2289, 2006, doi: 10.1002/elan.200603637.

[54] S. Venkatraman et al., "In Vitro and In Vivo Evaluation of PEDOT Microelectrodes for Neural Stimulation and Recording," vol. 19, no. 3, pp. 307-316, 2011.

[55] A. Petrossians et al., "Electrodeposition and Characterization of Thin-Film Platinum-Iridium Alloys for Biological Interfaces," J. Electrochem. Soc., vol. 158, no. 5, pp. D269-D276, 2011, doi: 10.1149/1.3578048.

[56] C. D. Lee, E. M. Hudak, J. J. Whalen, A. Petrossians, and J. D. Weiland, "Low-Impedance, High Surface Area Pt-Ir Electrodeposited on Cochlear Implant Electrodes," J. Electrochem. Soc., vol. 165, no. 12, pp. G3015-G3017, 2018, doi: 10.1149/2.0031812jes. 
[57] I. R. Cassar et al., "Electrodeposited platinum-iridium coating improves in vivo recording performance of chronically implanted microelectrode arrays," Biomaterials, vol. 205, no. November 2018, pp. 120-132, 2019, doi: 10.1016/j.biomaterials.2019.03.017.

[58] I. M. Taylor, E. M. Robbins, K. A. Catt, P. A. Cody, C. L. Happe, and X. T. Cui, "Enhanced dopamine detection sensitivity by PEDOT/graphene oxide coating on in vivo carbon fiber electrodes," Biosens. Bioelectron., vol. 89, 2017, doi: 10.1016/j.bios.2016.05.084.

[59] S. Mohanaraj et al., "Gold nanoparticle modified carbon fiber microelectrodes for enhanced neurochemical detection," J. Vis. Exp., vol. 2019, no. 147, 2019, doi: 10.3791/59552.

[60] B. Pusch, Jens, Wohlmann, "Chapter 2 - Carbon Fibers." . 